\title{
Visual quality and performance following bilateral implantation of TECNIS Symfony intraocular lenses with or without micro-monovision
}

This article was published in the following Dove Press journal:

Clinical Ophthalmology

\author{
Jilin $\operatorname{Tan}^{1,2}$ \\ Yan Qin $^{2}$ \\ Changpeng Wang ${ }^{2}$ \\ Shiman Yuan ${ }^{2}$ \\ Jian $\mathrm{Ye}^{\mathrm{I}}$
}

'Department of Ophthalmology, Army Medical Center of PLA, Daping Hospital, Army Medical University, Chongaing 400042, People's Republic of China; ${ }^{2}$ Cataract Department, Chongqing Aier Mega Eye Hospital, Chongqing 400060,

People's Republic of China
Correspondence: Jian Ye Department of Ophthalmology, Army Medical Center of PLA, Daping Hospital, Army Medical University, Chongqing 400042, People's Republic of China Tel +86I 3708385175

Email yejian1979@I63.com
Purpose: To evaluate the visual quality and performance of TECNIS Symfony intraocular lenses (IOLs) implanted with or without micro-monovision approach.

Setting: Chongqing Aier Mega Eye Hospital, Chongqing, China; Daping Hospital of Army Medical University, Chongqing, China.

Design: Prospective study.

Methods: The study comprised 70 cataract patients who had bilateral implantation of TECNIS Symfony extended range of vision IOLs, either with intended micro-monovision (monovision group: 35 patients) or with intended emmetropia (control group: 35 patients). Visual acuity, modulation transfer function (MTF), defocus curve, spectacle independence, and photic phenomena were analyzed 3 months postoperatively.

Results: There was no significant difference between the two groups in binocular uncorrected visual acuity at distance. Mean binocular uncorrected visual acuity at intermediate (monovision: $0.81 \mathrm{dec}$; control: $0.58 \mathrm{dec}$ ) and near (monovision: $0.59 \mathrm{dec}$; control: $0.30 \mathrm{dec}$ ) were significantly better in the monovision group. In the monovision group, the difference of MTF values between the dominant and the non-dominant eyes was not statistically significant and MTF values were within the normal range for both eyes. Spectacle independence at intermediate and near was statistically significantly higher in the monovision group compared to the control group. The incidence of photic phenomena was similar in both groups. Conclusion: Micro-monovision implantation of TECNIS Symfony IOLs provided a superior range of visual acuity from far to near and higher spectacle independence compared to a control group targeted for emmetropia.

Keywords: cataract, extended range of vision, defocus curve, spectacle independence, emmetropia, photic phenomena

\section{Introduction}

With the constant improvement of devices and techniques, cataract surgery, originally aimed to purely restore far vision, has evolved into refractive cataract surgery to further improve postoperative visual outcomes. Correcting presbyopia and achieving high-quality full-rangevision have become the greatest goals of modern cataract surgery. ${ }^{1-3}$ Today, presbyopia can be corrected by multifocal intraocular lenses (MIOLs). The optical design of diffractive MIOLs is based on light refraction and diffraction, and the magnitude of near addition mainly depends on the number of diffractive rings. A higher addition therefore leads to an increased incidence of photic phenomena such as glare and halos, resulting in decreased visual quality and 
insufficient visual acuity. ${ }^{3,4}$ Currently, various MIOLs still have some deficiencies in terms of presbyopia correction. Finding better concepts for MIOLs is therefore a major goal of current research. 5 The TECNIS Symfony extended range of vision (ERV) IOL (Johnson \& Johnson Vision, Santa Ana, CA, USA) is a new-generation presbyopiacorrecting IOL, which is supposed to offer an improved visual quality and less photic phenomena compared to former MIOLs. In addition, this lens is designed to provide a continuous range of functional vision from far to near distances. Clinical studies with TECNIS Symfony IOLs demonstrated excellent distance and intermediate visual outcomes but it was also reported that visual results were somewhat limited at near distances. ${ }^{7,8}$

The purpose of our study was to evaluate the visual quality and performance after bilateral implantation of TECNIS Symfony IOLs and to investigate whether there are benefits in applying micro-monovision.

\section{Patients and methods}

This prospective study included patients with bilateral agerelated cataract who were treated in our hospital from October 2016 to June 2018. The study was approved by the medical ethics committee of Chongqing Aier Mega eye hospital and conducted in accordance with the principles of the Declaration of Helsinki. Patients were divided into two groups: the monovision group and the control group (non-monovision group). IOL power was calculated using the Haigis formula. In the monovision group, TECNIS Symfony IOL power calculations were performed using a micro-monovision approach aiming for minimal residual myopia $(\approx-0.50 \mathrm{D})$ in the nondominant eye and emmetropia in the dominant eye. In the control group, emmetropia was considered as the target refraction for both eyes.

We enrolled patients according to the following inclusion criteria: bilateral age-related cataract, patients desiring spectacle independence, rational understanding of postoperative effects, length of axial oculi from 22 to $28 \mathrm{~mm}$, corneal spherical aberration of $\geq 0.27 \mu \mathrm{m}$, possibility to differentiate between dominant and non-dominant eye, lens nucleus hardness within Grade I-IV according to Emerry grading. Patients were excluded from the study if one of the following conditions were present: corneal astigmatism of $>1.5$ diopters (D), keratoconus, fundus lesions, unstable tear film before surgery affecting visual acuity, photopic pupil diameter $<2 \mathrm{~mm}$ or mesopic pupil diameter $>6 \mathrm{~mm}$, professional drivers and people who often drive at night, previous ocular surgery, angle alpha or angle kappa distance $>0.5 \mathrm{~mm}$, intraoperative complications (eg, posterior capsule rupture), and patients who are unable to cooperate in examinations or to attend the follow-up visits. All patients are informed of the surgical plan and assessment of the prognosis, possible complications during and after surgery, preoperative and postoperative precautions, and all patients are required to sign a written informed consent form before the operation. This study was approved by the ethics committee of Chongqing Aiermega Eye Hospital, and the procedure was in line with the Helsinki declaration.

All patients underwent femtosecond-laser-assisted phacoemulsification and TECNIS Symfony (Johnson \& Johnson Vision Surgical, Inc., Santa Ana, USA) IOL implantation by the same experienced surgeon using the LenSx femtosecond laser and the Centurion phacoemulsification system (Alcon Laboratories, Inc., USA). To correct a preoperative corneal astigmatism of $>0.75 \mathrm{D}$, femtosecond-laser assisted corneal incisions were made in 19 eyes.

Preoperative and postoperative examinations included ocular aberrometry (iTrace; Tracey Technologies Corp., Houston, TX, USA), tomography (Pentacam HR; Oculus Optikgeräte GmbH, Wetzlar, Germany), refraction (RT-5100; Nidek Co., Ltd., Gamagori, Japan), biometry (IOLMaster 500; Carl Zeiss Meditec AG, Jena, Germany), and optical coherence tomography (3 D OCT-1000; TOPCON, Tokyo, Japan). Three months after surgery, standard logarithmic visual acuity charts (Shanghai Yuejin Medical Optical Machinery Factory) were used to measure monocular and binocular uncorrected distance visual acuity (UDVA; $5 \mathrm{~m}$ ), uncorrected intermediate visual acuity (UIVA; $67 \mathrm{~cm}$ ), and uncorrected near visual acuity (UNVA; $33 \mathrm{~cm}$ ). Additionally, subjective refraction, corrected distance visual acuity, and uncorrected monocular and binocular defocus curves were measured from $+1.0 \mathrm{D}$ to $-4.0 \mathrm{D}$ (in $0.5 \mathrm{D}$ steps). Postoperative modulation transfer function (MTF) values were measured only in the monovision group. The visual function scale questionnaire for multifocal intraocular lenses was applied and spectacle independence was calculated. ${ }^{5}$

\section{Statistical analysis}

The SPSS 19.0 statistical software (IBM Corp., Armonk, NY) was used for data analysis. All values were expressed as mean $\pm \mathrm{SD}$. Normality of the data samples was evaluated using the Kolmogorov-Smirnov test. The paired $t$-test was used for comparisons between the preoperative and postoperative data within one group and the independent sample $t$ test was used for comparisons between the 2 groups. The 
chi-square test was applied to compare the outcomes of the patient questionnaire. For all statistical tests, a $P$-value of less than 0.05 was considered statistically significant.

\section{Results}

One hundred and forty eyes of 70 patients were included in the study and divided into 2 groups according to the target refraction: the monovision group ( 70 eyes of 35 patients) and the control group (non-monovision; 70 eyes of 35 patients).

Mean patient age was $67.97 \pm 17.1$ years (range $45-84$ years) in the monovision group and $68.76 \pm 19.5$ years (range 46-85 years) in the control group with no statistically significant difference between the groups $(P=0.378)$. More details of the preoperative data of the 2 groups are shown in Table 1.

The outcomes regarding uncorrected visual acuity at 3 months are shown in Table 2. There was no statistically significant difference in binocular UDVA between the groups $(P=0.905)$. In contrast, binocular intermediate and near visual acuity was statistically significantly better in the monovision group compared to the control group $(P=0.001)$.

Table I Basic preoperative patient information of both groups

\begin{tabular}{|l|l|l|l|}
\hline & $\begin{array}{l}\text { Monovision } \\
\text { group }\end{array}$ & $\begin{array}{l}\text { Control } \\
\text { group }\end{array}$ & $\boldsymbol{P}$ \\
\hline $\begin{array}{l}\text { Average corneal astigma- } \\
\text { tism (D) }\end{array}$ & $0.68 \pm 0.19$ & $0.67 \pm 0.27$ & 0.746 \\
$\begin{array}{l}\text { Average axial length (mm) } \\
\text { Average corneal kerato- } \\
\text { metry (D) }\end{array}$ & $24.44 \pm 1.49$ & $24.60 \pm 1.90$ & 0.673 \\
Average implanted IOL (D) & $18.09 \pm 4.63$ & $18.21 \pm 4.99$ & 0.910 \\
\hline
\end{tabular}

The mean target refraction and the mean postoperative spherical equivalent in both groups are summarized in Table 3.

The postoperative monocular and binocular defocus curves are shown in Figure 1-3.

Three months after surgery, mean MTF values (total eye, centered on the optic axis,range within $3 \mathrm{~mm}$ diameter) in the monovision group were $0.328 \pm 0.098$ for the dominant eyes and $0.313 \pm 0.095$ for the nondominant eyes $(P=0.875)$.

Table 4 shows the outcomes with regard to the postoperative spectacle independence.

For distance vision, all patients in both groups stated that they were totally free from glasses. In the case of intermediate and near vision, spectacle independence was significantly higher in the monovision group compared to the control group.

The incidence of postoperative photic phenomena was low (Table 5). Overall, 29 patients described photic phenomena like halos and glare especially at night but the symptoms were all classified as mild and had no impact on routine work and life. In both groups, we found 15 eyes (11 patients) that had an angle alpha of $>0.3 \mathrm{~mm}$ and 9 of these patients $(81.82 \%)$ described postoperative glare symptoms.

Shortly after surgery, a total of 5 patients ( 2 patients in the monovision group and 3 patients in the control group) reported difficulties with long-time reading and wearing glasses did not significantly improve their conditions. An examination showed that the symptoms were all related to unstable tear films after surgery and the symptoms could be relieved by the use of artificial tears.

Table 2 Uncorrected distance, intermediate, and near visual acuity (decimal) 3 months postoperatively

\begin{tabular}{|l|l|l|l|l|l|l|}
\hline \multirow{2}{*}{} & \multicolumn{2}{l}{ Monovision group } & \multicolumn{2}{l|}{ Control group } \\
\cline { 2 - 7 } & Dominant eye & Non-dominant eye & Binocular & Dominant eye & Non-dominant eye & Binocular \\
\hline UCDVA & $0.86 \pm 0.15$ & $0.69 \pm 0.19$ & $0.87 \pm 0.21$ & $0.85 \pm 0.16$ & $0.82 \pm 0.19$ & $0.88 \pm 0.18$ \\
UCIVA & $0.56 \pm 0.13$ & $0.82 \pm 0.17$ & $0.81 \pm 0.19^{*}$ & $0.57 \pm 0.12$ & $0.58 \pm 0.18$ & $0.58 \pm 0.14^{*}$ \\
UCNVA & $0.31 \pm 0.07$ & $0.60 \pm 0.15$ & $0.59 \pm 0.16^{*}$ & $0.30 \pm 0.09$ & $0.29 \pm 0.11$ & $0.30 \pm 0.13^{*}$ \\
\hline
\end{tabular}

Note: *Statistically significant difference between groups $(P<0.05)$

Table 3 Comparison of 3 months postoperative mean spherical equivalent and mean target refraction (D)

\begin{tabular}{|l|l|l|l|l|}
\hline & \multicolumn{2}{|l|}{ Monovision group } & \multicolumn{2}{l|}{ Control group } \\
\cline { 2 - 5 } & Dominant & Non-dominant & Dominant & Non-dominant \\
\hline Target refraction & $-0.06 \pm 0.04$ & $-0.57 \pm 0.18$ & $-0.05 \pm 0.05$ & $-0.11 \pm 0.07$ \\
Actual refraction & $-0.12 \pm 0.05$ & $-0.68 \pm 0.18$ & $-0.10 \pm 0.09$ & $-0.16 \pm 0.07$ \\
\hline
\end{tabular}




\section{3 months post-op defocus curve}

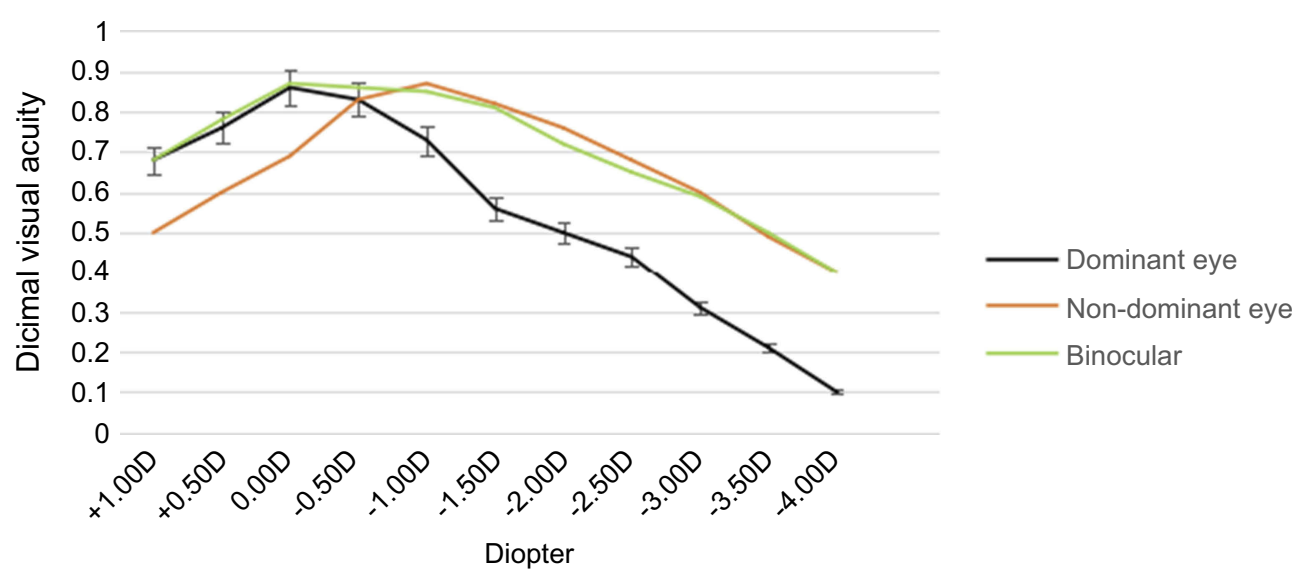

Figure I Monocular and binocular defocus curves in the monovision group 3 months postoperatively.

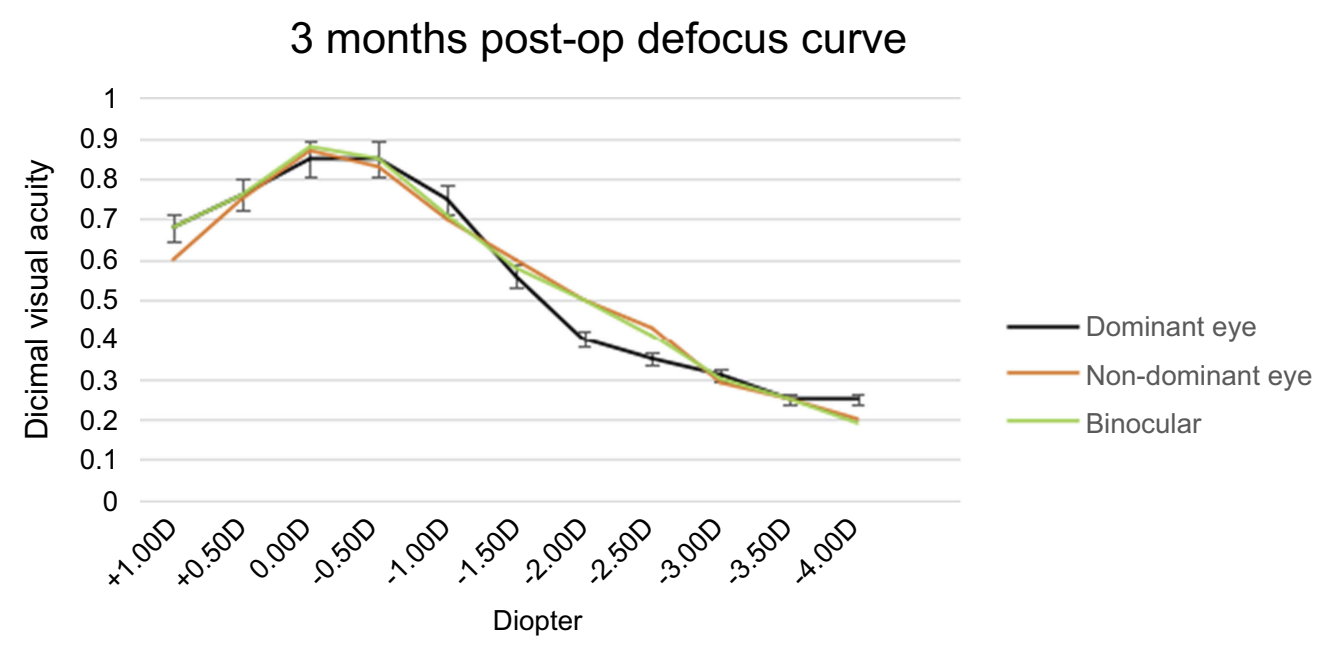

Figure 2 Monocular and binocular defocus curves in the control group 3 months postoperatively.

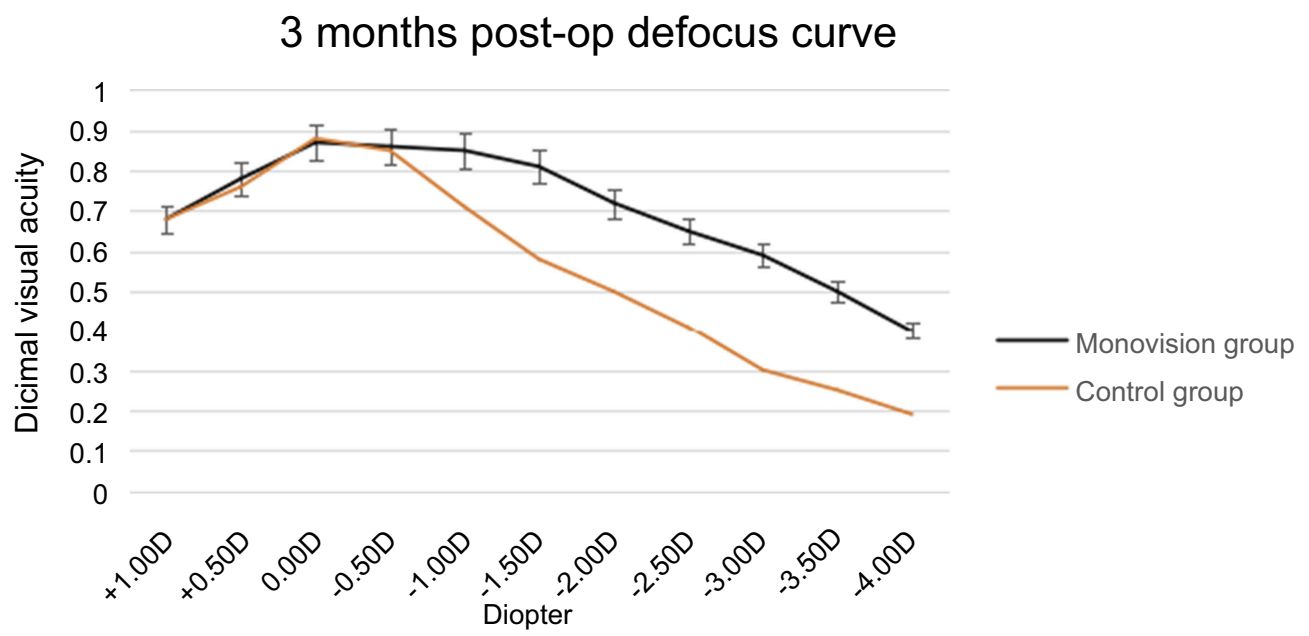

Figure 3 Comparison of binocular defocus curves in the monovision group and the control group 3 months postoperatively. 
Table 4 Postoperative spectacle independence in both groups

\begin{tabular}{|l|l|l|l|}
\hline & $\begin{array}{l}\text { Monovision } \\
\text { group }\end{array}$ & $\begin{array}{l}\text { Control } \\
\text { group }\end{array}$ & $\boldsymbol{P}$ \\
\hline Distance, n (\%) & $35(100)$ & $35(100)$ & - \\
Intermediate, n (\%) & $35(100)$ & $31(88.57)$ & 0.039 \\
Near, n (\%) & $31(88.57)$ & $10(28.57)$ & 0.001 \\
\hline
\end{tabular}

Table 5 Incidence of photic phenomena 3 months after surgery

\begin{tabular}{|l|l|l|l|}
\hline & $\begin{array}{l}\text { Monovision } \\
\text { group }\end{array}$ & $\begin{array}{l}\text { Control } \\
\text { group }\end{array}$ & $\boldsymbol{P}$ \\
\hline Halo, n (\%) & $15(42.86)$ & $14(40.00)$ & 0.808 \\
Glare, n (\%) & $7(20.00)$ & $6(17.14)$ & 0.759 \\
Starburst, n (\%) & $2(5.71)$ & $3(8.57)$ & 0.643 \\
Double images, n (\%) & $0(0.00)$ & $0(0.00)$ & - \\
\hline
\end{tabular}

\section{Discussion}

Currently, there are mainly two ways to correct presbyopia during cataract surgery. Firstly, monovision can be applied using monofocal IOLs. Here, the dominant eye is normally targeted for emmetropia and the non-dominant eye is targeted for myopia (from $-1.50 \mathrm{D}$ to $-3.0 \mathrm{D}$ ). The second way is the implantation of MIOLs.

Monovision means that one eye is corrected for distance and the second eye for near. The visual system allows the blurred image to be selectively suppressed and the clear image of the other eye to be accepted in order to see clearly at far and near distances. Monovision was first proposed by Westsmith in 1958 when they developed contact lenses for presbyopia correction. ${ }^{9}$ In 1984, Boerner reported on the application of monovision with IOLs and their satisfactory clinical results. ${ }^{10}$ This was confirmed by Greenbaum in 2002 who also reported about good outcomes in cataract patients with high ametropia. ${ }^{11}$ Monovision with monofocal IOLs can improve near visual acuity. Depending on the level of artificially generated anisometropia; however, this can have a negative effect on visual function, such as stereopsis, contrast sensitivity, and the visual field.

Another way to correct presbyopia is the use of MIOLs, currently available in different optic designs like refractive, diffractive, or hybrid MIOLs. MIOLs can improve near vision, but can also reduce contrast sensitivity and cause photic phenomena. ${ }^{12}$ For diffractive MIOLs, the amount of near addition is mainly determined by the number of diffractive rings. For example, the Restor $+4.0 \mathrm{D}$ MIOL provided good near vision but relatively high photic phenomena such as halos and glare. ${ }^{13,14}$ In contrast, the Restor $+2.5 \mathrm{D}$ MIOL offered better visual acuity at far and intermediate distances with relatively less optical phenomena but shows unsatisfactory near vision. ${ }^{15}$ The TECNIS Symfony ERV IOL was developed to fully meet patients' requirements regarding a continuous clear range of vision from far to near with less photic phenomena.The results of our study show that patients of the control group without micro-monovision achieved good distance and intermediate visual acuity but relatively poor near vision with a near-vision spectacle independence of only $28.57 \%$ and a lower satisfaction rate than reported elsewhere. ${ }^{16,17}$ It is estimated that this is related to a different patient population (eg, race and height) and therefore different reading distance requirements. In general, the reading distance is greater in Europe and in the US $(40 \mathrm{~cm})$ than in China $(33 \mathrm{~cm})$.

The results of our study showed that the micro-monovision approach has no significant impact on binocular UDVA. In the monovision group, UNVA and UIVA of the non-dominant eyes were $0.60 \pm 0.15(\mathrm{dec})$ and $0.82 \pm 0.17$ (dec), respectively, which were higher compared to the dominant eyes. Binocular UDVA was $0.87 \pm 0.21(\mathrm{dec})$ in the monovision group and 0.88 \pm 0.18 (dec) in the control group without a statistically significant difference. However, in the monovision group, binocular UIVA and UNVA were $0.81 \pm 0.19$ (dec) and $0.59 \pm 0.16$ (dec), respectively, which were statistically significantly higher than in the control group (UIVA: $0.58 \pm 0.14$ [dec]; UNVA: 0.30 \pm 0.13 [dec]). In the monovision group, the binocular UIVA and the UNVA could thus be improved without affecting the binocular UDVA.

The defocus curve of TECNIS Symfony IOLs presented a slowly descending unimodal course. In the monovision group, the monocular defocus curve of the non-dominant eyes with residual myopia was shifted to the right at about 0.5 to $-0.75 \mathrm{D}$ but the shape was comparable to that of the dominant eyes. MTF values in the monovision group were within the normal range $(>0.300)$ for both the dominant eyes and non-dominant eyes without a statistically significant difference between them. The defocus capacity was about $2.5 \mathrm{D}$ for a visual acuity level of at least $0.5(\mathrm{dec})$, and about $1.25 \mathrm{D}$ for a visual acuity level of at least $0.8(\mathrm{dec})$ for the dominant and the non-dominant eyes. Binocularly, the monovision group adapted to the micro-monovision approach and the shape of the defocus curve changed considerably. The defocus capacity reached about $3.0 \mathrm{D}$ for a visual acuity level of at least 0.5 (dec) and about 1.75 D for a visual acuity of at least $0.8(\mathrm{dec})$. Binocularly, reading visual acuity reached 0.5 (dec) at $33 \mathrm{~cm}$ and visual acuity was at least $0.5(\mathrm{dec})$ for distance, intermediate, and near vision. The binocular defocus curve of the monovision group showed no wave bottom 
with a very gradual decline. In addition, distance and intermediate visual acuity was at least 0.8 (dec) which is comparable to the results of other studies. ${ }^{18}$

In the control group, there was no difference between the monocular and the binocular defocus curve and the binocular UNVA and UIVA visual acuity was not significantly improved compared to the monocular results. Nearvision spectacle independence was statistically significantly higher in the monovision group (88.57\%) compared to the control group (28.57\%).

In this study, the 3-month results also showed that in the monovision group mean UDVA was $0.69 \pm 0.19$ for the non-dominant eyes, which was apparently lower than for the dominant eyes $(0.86 \pm 0.15)$ and for the control group $(0.85 \pm 0.16$ and $0.82 \pm 0.18$, respectively). It indicated that this depth of focus of the TECNIS Symfony intraocular lens did not mean that the visual acuity did not decrease, but was a parabolic curve with the focus as the peak and with both sides decreasing relatively gradually. Therefore, even within the range of $1.5 \mathrm{D}$ depth of focus of this intraocular lens, the UDVA would still decrease with the increase of the target refraction, but would decrease slowly. The course of the defocus curve and the downtrend also further proved such change.

Postoperatively, a small number of patients in both groups developed asthenopia but the symptoms could be relieved with the use of artificial tears which indicated that this was mainly related to postoperative xerophthalmia and not caused by binocular micro-monovision. It has been reported that tear film disorders can be caused by the application of femtosecond lasers and medication in the perioperative period. ${ }^{19}$ Overall, $42.86 \%$ of patients in the monovision group and $40.0 \%$ of patients in the control group described postoperative halos at night. The symptom, however, was classified as very mild without affecting routine work and life. Seven patients $(20.0 \%)$ in the monovision group and 6 patients $(17.14 \%)$ in the control group described glare symptoms but their visual acuity was good. We suggest that the glare symptoms were mainly caused by a large angle alpha (the angle between the optical axis and the visual axis). In our study, a total of 11 patients (15 eyes) showed an angle alpha distance of $>0.3 \mathrm{~mm}$ and 9 of those 11 patients $(81.82 \%)$ described postoperative glare symptoms. However, visual acuity in these patients was not affected. An explanation for this might be that the visual axis of the eyes was not interfered by the diffractive steps, since the central diffractive ring of the TECNIS Symfony IOL has a large diameter of $1.6 \mathrm{~mm}$. A big alpha angle has the same effect as an IOL decentration. The TECNIS Symfony IOL has a negative spherical aberration of -0.27 $\mu \mathrm{m}$ and for an intraocular lens with a high negative spherical aberration, a big alpha angle would decrease the visual quality and increase visual interference such as glare. ${ }^{20}$

\section{Conclusion}

The TECNIS Symfony ERV IOL provided successful visual restoration after cataract surgery. Targeting for micro-monovision in the non-dominant eye did not affect binocular distance visual acuity and provided better intermediate and near visual acuity and higher spectacle independence compared to the emmetropic control group. Attention should be paid to specific populations and to the impact of angle alpha on visual quality at night.

\section{Acknowledgment}

This work was made possible by the funding of former study "Clinical studies of femtosecend laser-assisted cataract surgery combinated multifocal intraocular lenses in correcting presbyopia" (AF152D10).

\section{Disclosure}

Dr Tan reports grants from Aier Eye Group Co. Ltd, during the conduct of the study outside the submitted work. The authors report no other conflicts of interest in this work.

\section{References}

1. Gatinel D, Loicq J. Clinically relevant optical properties of bifocal, trifocal, and extended depth of focus intraocular lenses. Refract Surg. 2016;32(4):273-280. doi:10.3928/1081597X-20160121-07

2. Zhaohui L, Ye Z, Yang H. "Multiple focus" problem with multifocal intraocular lens. Chin J Ophthal. 2017;53(4):244-248.

3. Li L, Guangying Z, Yating Z, Yun L. Clinical observation of long-term visual quality after implantation of aspheric multifocal intraocular lens. Chin J Ophthal. 2017;53(8):599-609.

4. Yune Z, Nvxia T. Measurement of distance, intermediate and near visual acuity in early stage after implantation of ReSTOR multifocal intraocular lens. Chin J Ophthal. 2008;44(1):33-37.

5. Yan Q, Jilin T, Xiaohong Z, et al. Assessment of visual quality after femtosecond-laser-assisted cataract surgery in combination with binocular implantation of Restor+2.5 D and Restor+3.0 D multifocal intraocular lenses. Chin J Ophthal. 2017;7(5):203-210.

6. Breyer DRH, Kaymak H, Ax T, et al. Multifocal intraocular lenses and extended depth of focus intraocular lensest. Asia Pac J Ophthalmol (Phila). 2017;6(4):339-349.

7. Kaymak H, Hohn F, Breyer DR, et al. Functional results 3 months after implantation of an "extended range of vision" intraocular lens. Klin Monbl Augenheilkd. 2016;233(8):923-927.

8. Shasha X, Guiqiu Z, Lin L, Xiaoni Y, Jing L, Cui L. Comparative study of visual quality of patients after Symfony and multifocal intraocular lens implantation. J Precis Med. 2018;33(2):166-168. 
9. Harris MG, Classe JG. Clinicolegal considerations of monovision. Am Optom Assoc. 1988;59:491-495.

10. Boerner CF, Thrasher BH. Results of monovision correction in bilateral pseudophakes. Am Intraocul Implant Soc. 1984;10:49-50. doi:10.1016/S0146-2776(84)80077-4

11. Greenbaum S. Monovision pseudophakia. J Cataract Refract Surg. 2002;28:1439-1443. doi:10.1016/S0886-3350(02)01218-X

12. De Vries NE, Nuijts RM. Multifocal intraocular lenses in cataract surgery: literature review of benefits and side effects. $J$ Cataract Refract Surg. 2013;39(2):268-278. doi:10.1016/j.jcrs.2012.12.002

13. Attia MS, Khoramnia R, Auffarth GU, et al. Near and intermediate visual and reading performance of patients with a multifocal apodized diffractive intraocular lens using an electronic reading desk. $J$ Cataract Refract Surg. 2016;42(4):582-590. doi:10.1016/j. jers.2015.11.047.

14. Nakamura K, Bissen-Miyajima H, Yoshino M, Oki S. Visual function after contralateral implantation of multifocal intraocular lenses with +3.0 and +4.0 diopter additions. Asia Pac J Ophthalmol (Phila). 2015;4(6):329-333. doi:10.1097/APO.

15. Vilar C, Hida WT, de Medeiros AL, et al. Comparison between bilateral implantation of a trifocal intraocular lens and blended implantation of two bifocal intraocular lenses. Clin Ophthalmol. 2017;11:1393-1397. doi:10.2147/OPTH.S139909.
16. Cochener B, Concerto G. Clinical outcomes of a new extended range of vision intraocular lens: international multicenter concerto study. $J$ Cataract Refract Surg. 2016;42(9):1268-1275. doi:10.1016/j. jcrs.2016.06.033

17. Emilio P, Francesco C, Francesco A, et al. Comparative analysis of visual outcomes with 4 intraocular lenses: monofocal, multifocal, and extended range of vision. J Cataract Refract Surg. 2018;44 (2):156-167. doi:10.1016/j.jcrs.2017.11.011

18. Domínguez-Vicent A, Esteve-Taboada JJ, Del Águila-Carrasco AJ, Ferrer-Blasco T, Montés-Micó R. In vitro optical quality comparison between the mini WELL ready progressive multifocal and the TECNIS Symfony. Graefes Arch Clin Exp Ophthalmol = Albrecht Von Graefes Archiv Fur Klinische Und Experimentelle Ophthalmologie. 2016;254 (7):1387-1397. doi:10.1007/s00417-015-3240-7

19. Yao K, Yu Y. New understanding of attaching importance to femtosecond-laser-assisted cataract surgery. Chin J Ophthal. 2017;7 (3):97-102.

20. Karhanová M, Pluháček F, Mlčák $P$, et al. The importance of angle kappa evaluation for implantation of diffractive multifocal intra-ocular lenses using pseudophakic eye model. Aca Ophthalmol. 2015;93(2):e123-e128.

\section{Publish your work in this journal}

Clinical Ophthalmology is an international, peer-reviewed journal covering all subspecialties within ophthalmology. Key topics include: Optometry; Visual science; Pharmacology and drug therapy in eye diseases; Basic Sciences; Primary and Secondary eye care; Patient Safety and Quality of Care Improvements. This journal is indexed on PubMed
Central and CAS, and is the official journal of The Society of Clinical Ophthalmology (SCO). The manuscript management system is completely online and includes a very quick and fair peer-review system, which is all easy to use. Visit http://www.dovepress.com/ testimonials.php to read real quotes from published authors. 